\title{
Novel mouse model of steroid-resistant allergic rhinitis by repeated intranasal administration of OVA
}

\author{
Kazunari Izumi*, Hideki Kawasome, Atsushi Azuma, Kenjiro Sasaki, Masayuki Kamada, Kazushi Sakurai \\ From 3rd WAO International Scientific Conference (WISC) 2014 \\ Rio de Janeiro, Brazil. 6-9 December 2014
}

\section{Background}

Allergic rhinitis (AR) is one of uncontrollable inflammation diseases by a corticosteroid administration. Thus an ideal AR animal model had been required in this research field in order to develop a new effective remedy. We report our establishment of the novel AR animal model resistant to nasal corticosteroid.

\section{Methods}

$\mathrm{BALB} / \mathrm{c}$ mice were sensitized by intraperitoneal injection of ovalbumin (OVA) /ALUM. Two weeks after the sensitization, the mice were administrated OVA intranasally for five times a week (One-week-challenge arm), or five days in the first week followed by three times a week for every two days from the second to the fifth week (Fiveweek-challenge arm). In the both arms, the day before the first challenge, the mice were randomly divided into four groups $(\mathrm{n}=8)$ : negative control $(\mathrm{NC})$, vehicle control (VC), mometasone furoate (MF), and dexamethasone (DEX). For the NC groups, sensitized mice were intranasally treated with saline instead of OVA. In the MF groups, $5 \mu \mathrm{g} / 10 \mu \mathrm{L}$ of MF was administrated intranasally 30 minutes before the last OVA challenge. In the DEX groups, $1 \mathrm{mg} / \mathrm{kg}$ of DEX was administrated orally 1 hour before the last OVA challenge. The nasal congestion in early phase and late phase responses were measured by two-chambered, double-flow plethysmograph system as specific air way resistance (sRAW) 10 minutes and 3 hours after the last OVA challenge, respectively. The mice were sacrificed by $\mathrm{CO}_{2}$ gas inhalation and the nasal paraffin sections were made for histological evaluation.

\section{Results}

In the One-week-challenge arm, the sRAW in MF and DEX group were lower than $\mathrm{VC}$ group both in the early (inhibition rates: MF 73\%, DEX 94\%) and the late phase responses (MF 76\%, DEX 84\% inhibition). In the Fiveweeks-challenge arm, MF and DEX reduced the nasal congestion in the early phase (MF $42 \%$, DEX $58 \%$ inhibition), however, the inhibition rate of the late phase was significantly decreased in the MF group (MF 28\%, DEX $50 \%$ inhibition). The epithelial hyperplasia and the inflammatory cells infiltration were observed in the nasal mucosa, and these grades were more remarkable for Five-week-challenge arm than One-week-challenge arm.

\section{Conclusions}

After five weeks OVA challenge, the nasal congestion remained partially even after the nasal corticosteroid treatment. This repeated OVA administration method would provide a quite useful novel disease model to elucidate the mechanisms of nasal steroid-resistant-AR and develop a new remedy.

Published: 8 April 2015

doi:10.1186/1939-4551-8-S1-A30

Cite this article as: Izumi et al:: Novel mouse model of steroid-resistant allergic rhinitis by repeated intranasal administration of OVA. World Allergy Organization Journal 2015 8(Suppl 1):A30. 